\title{
A Comprehensive Survey On Power Saving Schemes (CSPSS) In IEEE 802.16E/M Networks
}

\author{
'Wisdom, D.D., ${ }^{2}$ Ajayi, E.A., ${ }^{3}$ Aladesote, O.I., \& ${ }^{4}$ Arinze, U.C. \\ 1 Department of Computer Science, Usmanu Danfodiyo University, Sokoto, Nigeria \\ ${ }^{2}$ Faculty of Information Science \& Technology. Multimedia University, Maleka, Malaysia. \\ 3Department of Computer Science, Federal Polytechnic, Lle-oluji, Nigeria \\ ${ }^{4}$ Department of Computer Science, University of Nigeria, Nsukka, Nigeria.
}

E-mails: 1danieldaudawisdom1@gmail.com; 2ebeseun@gmail.com; 3lomaladesote@yahoo.com; 4arinzechuchukwugmail.com

Phones: +2347030743902; +2347020396898; +2348030657156; +2348066256878

\begin{abstract}
Worldwide interoperability for micro wave access (WiMAX) is a wireless network that have attracted the attention of researchers on the area of power savings, because of the increase in multimedia applications that consumes more battery power of Mobile stations (MS). MS experience an increase in their daily operations with limited power enforced on the MS; hence the need to optimally increase efficiency; due to the excessive power consumption experienced by MS; which significantly affects the performance of MS since, MS are battery powered with a super impose life, improving the life time of MS is imperative. Thus, this has led to numerous research works on energy-savings. Hence, we have presented a survey on the recent and past energy-saving schemes, aimed at understanding some of the most relevant sources of inefficiency in energy savings and how some of these challenges are solved by the existing solutions. We further presented a comparative analysis on these schemes with the aim of identifying current challenges that are yet to be addressed by the research community as well as presents future directions towards efficient energy savings in WiMAX networks.
\end{abstract}

Keywords: Power-Savings, Consumption, WiMAX, Networks

Proceedings Reference Format

Wisdom, D.D., Ajayi, E.A.,3Aladesote, O.I., \& Arinze, U.C. (2021): A Comprehensive Survey On Power Saving Schemes (CSPSS) In IEEE 802.16E/M Networks. Proceedings of the 27th iSTEAMS Multidisciplinary Innovations \& Technology Transfer (MINTT) Conference. Academic City University College, Accra, Ghana. June, 2021. Pp 145-166 www.isteams.net/ghana2021. DOI - https://doi.org/ 10.22624/AIMS/ISTEAMS-2021/N27P14

\section{INTRODUCTION}

There have been rapid advancements in wireless communication in recent years. WiMAX networks as one of the growing wireless communication technologies has attracted attention around the world because of it economic and societal benefits. This has led the working group of the IEEE 802.16 to develop a series of WiMAX standards. The earlier standards of the IEEE 802.16-2004 specified the access between a base station (BS) and fixed Subscriber Stations (SSs) as fixed (IEEE, 2004); while in the subsequent standard, 
Mobile Stations (MSs) are supported by the IEEE 802.16e (IEEE, 2006). The IEEE 802.16-2009 (IEEE, 2009) consolidated the two standards and added additional mobility characteristics.

With another standard as the IEEE $802.16 \mathrm{~m}$ (IEEE, 2010), developed to provide the requirements for future International Mobile Telecommunications (IMT) Advanced networks with higher data rate and higher mobility as well. Because of the mobility feature added, prolonging the battery life of MSs became an important problem as well as one of the critical issues since MSs are battery powered with limited lifetime until recharging excise is conducted. As such, efforts have been made to design solutions that will minimize energy consumption with the aim of improving the efficiency of a mobile device with minimal response delay.

Several energy saving mechanisms have been proposed in (Hwang et al. 2010) (Eunju et al. 2009) (Jianbin et al. 2008) (Yang et al. 2013) (Shi et al. 2006) (Wisdom et al.2019) to improve the battery life of a MS. Most of these Mechanisms used Power-Saving Classes (PSC) of type I while a few of the Mechanisms used type II. The PSC Type I recommend for connections such as Best Effort (BE), Non-Real Time Services (NR-TS). Based on the standard, Power Saving Class becomes active at the frame specified as the start frame number for the first sleep windows (Saidu et al. 2017).

PSC Type II is recommended for connections of unsolicited grant services (UGS), such as real time variable rate (RT-VR) service connections. Unlike Type I, Type II uses constant sleep window size instead of doubling the sleep interval figure I. More so, contrary to Type I, at the listening windows of Type II the MS can send or receive any Service Data Units (SDUs) or their fragments, based on the standard (Saidu et al. 2017). While PSC of type III is used for managing multicast connections and operations as follows: In this paper, we have presented a survey on recent and past energy-saving schemes in WiMAX. The schemes operational mode, strengths and weaknesses are presented. A comparative analysis of these schemes is also presented to identify current challenges for future research. The rest of this research work is organized as follows. Section 2 presents the methodology used. Section 3 presents results and discussion while Section 4, concludes our study.

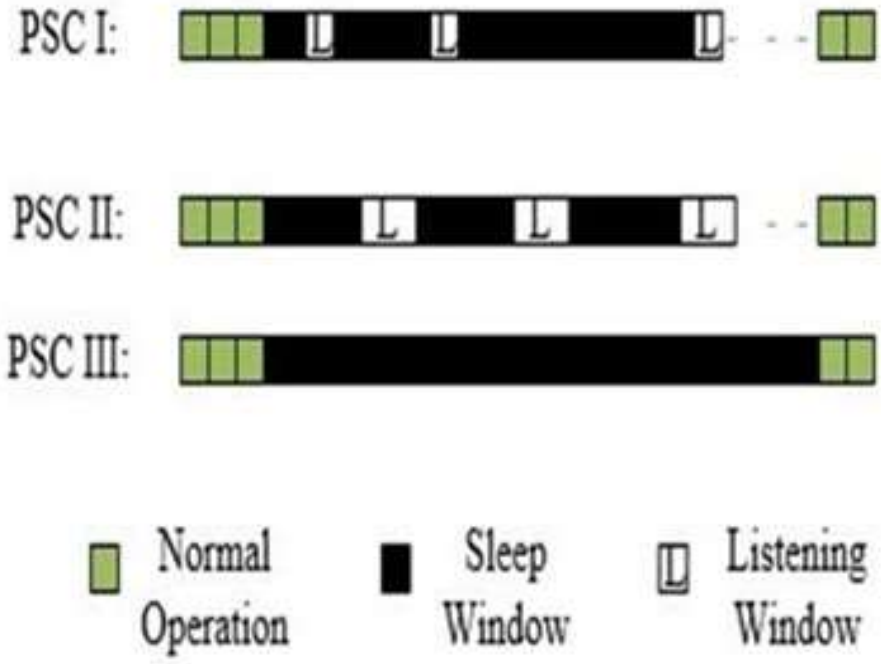

Figure 1. Shows Power Saving Classes (PSC) 
Table 1 : The Evolution of the IEEE 802.16 family

\begin{tabular}{|c|c|c|c|c|}
\hline & $802.16-2001$ & $802.16 a$ & $802.16-2004$ & $802.16 \mathrm{e}$ \\
\hline $\begin{array}{l}\text { Frequency } \\
\text { range }\end{array}$ & $10-16 \mathrm{GHz}$ & $2-11 \mathrm{GHz}$ & $2-11 \mathrm{GHz}$ & $2-6 \mathrm{GHz}$ \\
\hline $\begin{array}{l}\text { Channel } \\
\text { condition }\end{array}$ & Line-of-sight only & Nonline-of-sight & Nonline-of-sight & Nonline-of-sight \\
\hline $\begin{array}{l}\text { Channel } \\
\text { Bandwith }\end{array}$ & 20,25 and $28 \mathrm{MHz}$ & $1.25-28 \mathrm{MHz}$ & $1.25-28 \mathrm{MHz}$ & $1.25-20 \mathrm{MHz}$ \\
\hline $\begin{array}{l}\text { Modulation } \\
\text { scheme }\end{array}$ & $\begin{array}{l}\text { QPSK, 16QAM, } \\
\text { and 64QAM }\end{array}$ & $\begin{array}{l}\text { OFDM, QPSK, } \\
\text { 16QAM, and } \\
\text { 64QAM }\end{array}$ & $\begin{array}{c}\text { OFDM, QPSK, } \\
\text { 16QAM, and } \\
\text { 64QAM }\end{array}$ & $\begin{array}{c}\text { OFDMA,OFDM, QPSK, } \\
\text { 16QAM, and 64QAM }\end{array}$ \\
\hline Bit rate & 32-134Mbps & Up to 75 Mbps & Up to $75 \mathrm{Mbps}$ & Up to $15 \mathrm{Mbps}$ \\
\hline Mobility & Fixed & Fixed & Fixed & $\begin{array}{c}\text { Pedestrian mobility-regiona } \\
\text { roaming, maximum mobility } \\
\text { support: } 125 \mathrm{~km} / \mathrm{h}\end{array}$ \\
\hline $\begin{array}{l}\text { Typical cell } \\
\text { radius }\end{array}$ & 1-3 miles & $\begin{array}{c}\text { Maximum range } \\
\text { is } 30 \text { miles on } \\
\text { the basis of } \\
\text { antenna gain } \\
\text { and transmission } \\
\text { power }\end{array}$ & $\begin{array}{c}\text { Maximum range is } \\
30 \text { miles on the } \\
\text { basis of antenna } \\
\text { gain and } \\
\text { transmission } \\
\text { power }\end{array}$ & 1-3 miles \\
\hline Applications & $\begin{array}{c}\text { Replacement of } \\
\text { E1/T1 services for } \\
\text { enterprises, } \\
\text { backhaul for } \\
\text { hotspots, } \\
\text { residential } \\
\text { broadband access, } \\
\text { SOHO(small } \\
\text { office/home office) }\end{array}$ & $\begin{array}{c}\text { Alternative to } \\
\text { E1/T1, DSL, } \\
\text { cable backhaul } \\
\text { for cellular and } \\
\text { Wifi, VoIP, } \\
\text { internet } \\
\text { connections }\end{array}$ & $\begin{array}{c}\text { 802.16-2001 plus } \\
802.16 a \\
\text { applications }\end{array}$ & $\begin{array}{c}\text { 802.16-2004 applications } \\
\text { plus fixed VoIP, QoS-based } \\
\text { applications, and enterprise } \\
\text { networking }\end{array}$ \\
\hline
\end{tabular}

\section{METHODOLOGY}

In this section, we have presented an overview of WiMAX Networks and conducted an extensive review of both past and recent related literatures in WiMAX Networks in order to identify individual strength and weaknesses of each scheme, and presents possible future research directions as well as conducted a comparative study of the different power saving classes (PSC) and how their operational procedures, method of activation and deactivation are implemented and present an overview on WiMAX as well as promising issues yet on resolved as directions for further research work.

\subsection{Overview of WiMAX}

Worldwide Interoperability for Microwave Access (WiMAX) is an important wireless telecommunication technology in use. WiMAX as wireless metropolitan area network (WMAN) technology. 


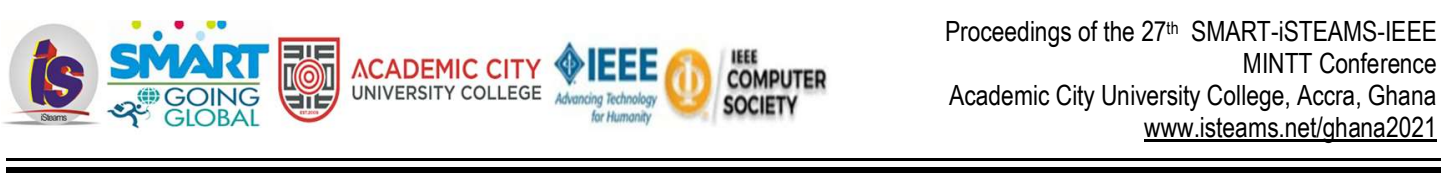

WiMAX is a wireless technology improved for the delivery of IP centric services over a wide area network (WAN); it's a scalable wireless platform used in the building of alternate and matching broadband networks. WiMAX structures are anticipated to deliver broadband access services to residential and enterprise customers in an economic manner. Lightly, WiMAX is a consistent wireless version of Ethernet made mainly as substitute to wire network such as cables modems DSL and T1/E1 Links to offer broadband access to premises.

In addition, WiMAX is a manufacturing organization formed by leading network communication, components, as well as apparatus companies to promote, certify compatibility as well as interoperability of broadband wireless kit that conforms to the IEEE 802.16 Standards. The IEEE802.16 medium access control (MAC) was design for point to multipoint broadband wireless access systems. The main duty of the MAC layer is to provide an interface such that the advanced transport layer as well as the physical layer can communicate each other. The MAC layer transmits data packets through the upper layer; these data packets are usually called MAC Service Data Units (MSDUs) and organize them into MAC Protocol Data Units (MPDUs) for broadcast over the air. For incoming broadcasts, the MAC layer does the reverse. The IEEE802.16-2004 and the IEEE802.16e-2005 MAC design includes a merging sub layer that can interface with a variety of advanced-layer protocols, such as ATM, TDM voice, Ethernet, IP, and any unknown future protocol.

The 802.16 MAC is design for Point to Multipoint (PMP) systems and is on Collision Sense Multiple Access with Collision Avoidance (CSMA/CA). More so, support for QoS is a essential part of the WiMAX MAC layer design; it takes some of the main ideas behind its QoS design from the Data over Cable Service Interface Specifications (DOCSIS) cable modem standard. A better QoS control can be achieved by a connectionoriented WiMAX MAC architecture, where all DL as well as UL connections are meticulously controlled through serving Base Stations (BS). WiMAX also explains the concept of a services flow. A services flow is a unidirectional flow of packets with a particular set of QoS restrictions identified by a Service Flow Identified (SFID). The physical layer is according to Orthogonal Frequency Division Multiplexing (OFDM). It is through the broadcast scheme of choice to enable high speed data, video and multimedia communications and is used by a variety of commercial broadband networks, which includes DSL, Wi-Fi, Digital Video Broadcasthandheld (DVB-H), and media Flo. WiMAX OFDM is a sophisticated and well-organized structure for high data rate broadcast in a non-line-of-sight or multipath radio environment.

The physical layer of WiMAX is relatively flexible therefore; the data rate performance differs based on the operating parameters. Parameters that have a substantial impact on the physical layer data rate are channel bandwidth, the modulation as well as coding scheme used. Other parameters, as number of sub-channels, OFDM guard time, and oversampling rate, have an impact as well. WiMAX operates as Wi-Fi, but advanced in speed over greater distances and used by numbers of handlers. It also has the ability to provide services even in locations that are difficult for wired networks to influence and the ability to overcome the physical limitations of outdated wired network. WiMAX is projected to offer initially up to about 40 mbps capacity per wireless channel for both fixed and portable networks, depending on the particular technical configuration chosen, enough to support hundreds of businesses with T-1 speed connectivity and thousands of residences with DSL speed connectivity. WiMAX can support voice, video and internet data. WiMAX provides wireless access to buildings, either in competition to existing wired networks or alone in currently un-served rural or less populated places. WiMAX is also used to connect WLAN hotspots to the internet. 


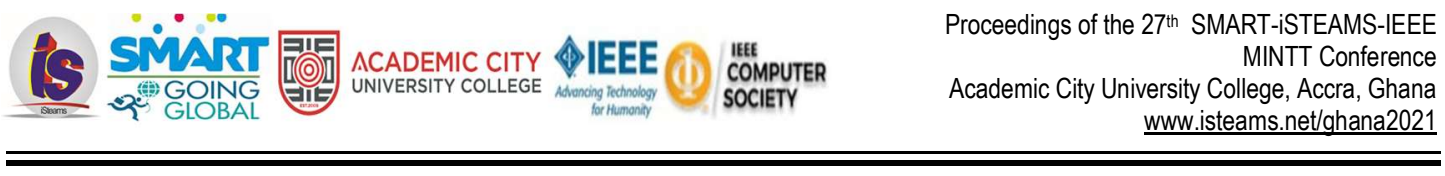

It can also provide broadband connectivity to mobile devices, although it may not be as fast as in the fixed networks, but expectations are for about $15 \mathrm{mbps}$ capacity in a $3 \mathrm{~km}$ cell coverage range. With WiMAX, operators can access and go online at broadband speeds, virtually all the time they wish to, from within a metro zone. Finally, WiMAX network can be installed in a variety of spectrum bands: $2.3 \mathrm{GHZ}, 2.5 \mathrm{GHZ}$, $3.5 \mathrm{GHZ}$, and $5.8 \mathrm{GHZ}$ respectively.

\subsection{Survey on Energy Saving Schemes}

A survey on existing energy schemes is presented in this section. The schemes are studied by highlighting their operational procedure, strengths as well as weaknesses of each scheme. In addition, the survey is further subdivided into 3 subgroups namely power saving mechanisms, adaptive power saving mechanisms and dynamic power saving mechanisms for better understanding and readability as follows:

\subsection{Power Saving Mechanisms}

In (Hwang et al. 2010), an energy scheme with periodic traffic indications was proposed to decrease energy consumption. The scheme tries to decrease the state transition overhead for both wake as well as sleep modes, hence decreasing the increase delay for state transition as well as improving energy saving efficiency. The mechanism reduced the average rate of consumption of the MS while improving the quality of service (QoS), but suffers little increase in delay. In Eunju et al.2009, a mechanism with binary exponential traffic indications message was proposed in order to reduce consumption rate. This mechanism omits MOBSLP-REQ/RSP messages with a traffic indication (TRF-IND) interval as an important parameter; the mechanism applies the truncated binary exponential increase approach for its length and tries to reduce the scheme overhead for the state transition for both wake as well as sleep intervals, thereby minimizing the delay due to its transition and significantly improve efficiency. The mechanism consumes less energy compared to PSC of type I standard. However, the scheme derivations are intricate that extending the research is an important concern. In Eunju et al.2007, power mechanism with periodic traffic indication was introduced to improve efficiency. The mechanism used traffic indication (TRF-IND) messages to initiate data transfer at each constant time.

The TRF-IND messages consist of a listening/wake interval as well as sleep interval. at the listening interval a MS synchronizes with it present BS and decides to switch to wake-mode or remain in a sleep-mode. If there exist traffics in the buffer for the tagged MS the BS sends a positive TRF-IND message and the MS switch to wake-mode. The BS sends data during the wake-state thus end transmission if no traffic arrival during a time-out/fixed time of a constant length $\mathrm{T}$. If any data traffic arrives during the close-down time $\mathrm{T}$, the BS keeps on the wake state and transmits the data. Else, the MS goes to a sleep-mode from the wake state without exchanging MOB-SLP-REQ/RSP messages. The mechanism decreased the rate of consumption while satisfying the QoS. However, it ignores considering up-link traffics.

In (Eunmi et al. 2015), a remaining energy-aware quality-of-service (QoS) based binary-exponential sleep mode (BE-SM) scheme was proposed to minimize consumption rate of MS. The proposed BE-SM allocates the sleep cycles as well as enhanced the frequency of LoRE reports while reducing the consumption rate as well as determine a considerable QoS based on the remaining energy and subjective quality degradation. However, it fails to consider the average waiting time. In (Almhana et al. 2008), an online Exponential Mixture (EM) algorithm was introduced to fit the exponential distributions to an appropriate inactive time. The algorithm begins when a MS has no traffic to transit; the MS sends a MOB-SLP-REQ message to the BS which includes the duration of the sleep period. 


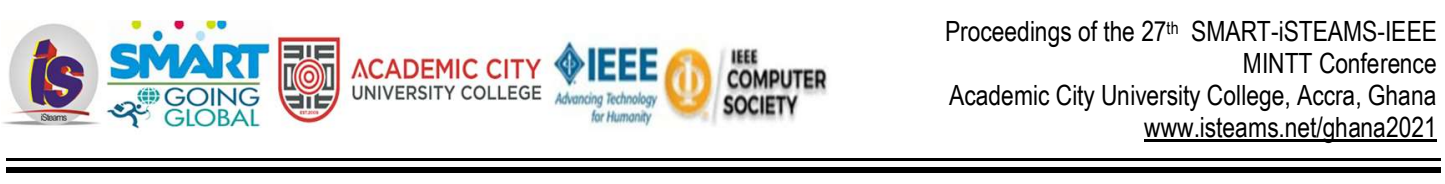

When the request is granted, the MS switch to sleep mode by turning off its receiver for a dedicated sleep interval. When new frames arrives during the sleep interval of the MS, the BS buffers the arriving frames and sets the traffic indicator (TRF-IND) to 1 in order to update the MS. When the sleep interval times out the MS wakes up and listens to the signaling channel for a dedicated period of time known as the listening period. If its TRF-IND is set to 1 it switches to active mode. Otherwise, it doubles its sleep interval and reenters sleep mode. Once there is no TRF-IND message, the MS initializes the first sleep interval with the minimal duration Tmin, and then doubled the sleep interval until a maximum value Tmax is attained. The algorithm improved power efficiency but ignores developing algorithms that can adapt the power savings for time-varying traffic which may results to waste of energy.

In (Xiao et al. 2006), an Enhanced Energy Saving Mechanism (EESM) was proposed to address the Excessive listening operations of ESM which guzzle much energy when traffic is low with high consumption rate. In the proposed scheme, a MS used half of the last sleep session after terminating from the previous sleep-session as the initiate sleep interval in next sleep-session if the initiate sleep interval is less than Tmin, the initiate sleep interval is equal to Tmin. If traffic is low the SDU arrival is set bulky enough such that the proposed EESM can effectively reduce the number of listening intervals in a sleep-session. It improved the battery life of MS efficiently. However, it fails to consider RT-VR traffics which may further improve battery life of MS. In [20], a Scheduled Power-Saving Mechanism (SPSM) was introduced to schedule sleep intervals operations for connections that newly initiate sleep-mode by controlling the main operation mode in PSC, namely: Tmin and Tmax, as well as the initiation time of sleep session of low QoS connections enabling the sum duration of the available state to decrease from $16 \mathrm{U}$ to $13 \mathrm{U}$. The proposed scheme improved the battery life by increasing the engaged time during with connections of MS in sleep-state. However, there is an increase delay process.

In (Kim et al.2009), Enhanced Mechanism (EPSM) was proposed to adaptively manage the sleep parameters, efficiently reflecting the sleep duration of MS. This mechanism comprises of two conditions $X$ and $Y$ which takes into action the remaining energy state: $X$ is for advanced energy conservation with average delay; $Y$ is for more demanding energy conservation that keeps longer sleep session. The mechanism enhances efficiency at interval periodically for a short time to check for DL traffics and decides to switch to wake/continue to sleep. The scheme sleep intervals increases exponentially when no arrival of traffics, the mechanism significantly saved energy with an increase in response delay. In (Zhang et al.2009), an Enhanced Energy Management Mechanism (EEMM) was proposed to expand the battery life of MS. The proposed EEMM provides great performance with a tradeoff, adaptively adjusting the sleep intervals size and significantly reduce consumption. Nevertheless, the EEMM scheme enhanced the battery life with an in appropriate choose of sleep parameters.

In (Wong et al. 2009), a real-time heuristic algorithm (RTHA) was proposed to reduce the switching frequency of MS. The scheme negotiates at each listening session after the total sleeping sessions. According to the embedded information in the MOB-TRF-IND message, such as traffic indicator, Packet arrival rate at the BS buffer $(\lambda)$ and Current buffer occupancy at the BS (i), the MS decides and wakes up to full normal operational mode and receives frames waiting at the BS. The MS continue to sleep with negative indication. The MS then makes the decision to run the algorithm/not. The proposed algorithm three criteria are computed and evaluated. The algorithm performed better with average waiting time kwon as response delay which subsequently results in poor QoS. In (Saidu et al.2015), an Efficient Battery Life-Aware Power Saving scheme (EBLAPS) was introduced to reduce the average delay. 


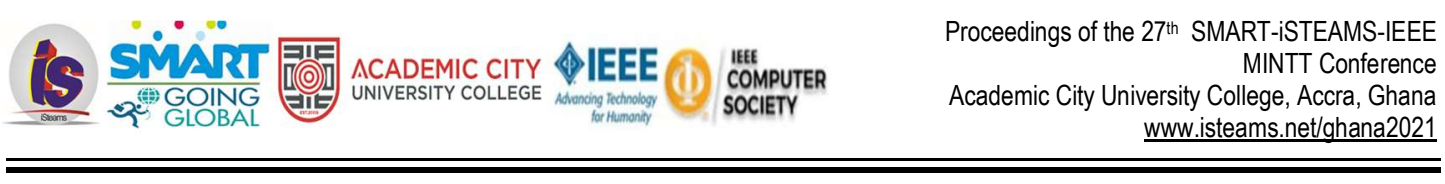

The Scheme adaptively tunes the sleep parameters according to the traffic arrival. The schemes reduced the frequent transition to listening session as well as the rate of MS consumption with a longer sleep session. In (Nga et al.2007), a Delay-Guaranteed Energy Saving (DGES) scheme was proposed to decrease the consumption rate of MS; the scheme tries to obtain an optimal (Tmax) value with fixed Tmin value. The scheme insured the required response delay by obtaining an optimal value for a fixed Tmin value. The scheme assumed that the Tmin is determined as well as $L$ is fixed. It effectively improve the battery life of MS, with an increase in consumption. In (Wong et al. 2009), joint optimal timeout scheme was proposed to minimize switching cost of MS. The scheme comprises of three main components, namely: optimal timeout value, conditional probability of MS energy savings. The scheme runs incessantly on wake interval until it becomes idle, while an optimal timeout value is obtained.

The scheme switches to a timeout period using optimal timeout function. When traffics are not detected, the scheme decides type I/II base on it designed conditional probability. The joint scheme enables MS to be aware when to sleep and how to sleep with less switching cost, with small increase in delay which may lead to energy waste. In (Liao et al.2016), a scheduling algorithm for multiple MS was proposed to schedule multiple MS with UGS connections so that the QoS requirement of each MS can be satisfied after scheduling. The scheme prevents occurrence of interference in MSs and tries to meet the QoS requirement of the MS after scheduling. It achieves higher bandwidth utilization with increase in signaling overhead which lead in poor QoS. In (Kong et al.2009), a theoretical framework based on the semi-Markov Decision Process (MDP) was proposed to maximize in-efficiency of MS with QoS guarantee.

The MDP model is a transitional diagram where each state represents one unique sleep stage. Based on this proposed scheme, the model is represented in three state, normal mode sleep mode of type I and II with a number of policy (denoted as $\mathrm{R}$ ) for controlling the operational mode. $\mathrm{R}$ is defined as a prescription for taking actions at each decision epoch whether to be a deterministic policy/randomized policy. The scheme achieved higher efficiency with an increase in response time. In (Yang et al. 2013), integrated loadbased power saving for was proposed for better efficiency. In the first strategy of the proposed scheme, S1, allows BS to switch to sleep mode when all MS are in the sleep mode. The Power Saving Efficiency (PSE) at the BS is limited by traffic load and sleep schedule. The second strategy considers that power saving at BS is more often than not better than power saving at MS.

Hence, a threshold for power saving (PSE_TH) at BS is set earlier in the second strategy S2, where the sleep scheduling algorithm in LBPS schemes must be revised in other to integrate BS and meet the requirement of PSE_TH. The scheme achieves high efficiency for BS and MS. with no balancing between power-saving and the increase response delay. In (Azad et al. 2011), an optimal control of sleep periods was proposed to optimally schedule shutting off its transceiver to minimize consumption. The proposed scheme terminal undergoes a sequence of sleep and listening session until an active request is detected. From the beginning of each sleep session the MS choose sleep session length. The scheme reduced the listening session. With no consideration to utilize renewal theory that captures the effects of remaining interarrival of the MS.

In (Chen and Tsao, 2006), two algorithms were proposed: periodic on-off scheme (PS) and aperiodic on off scheme (AS) to fully utilize the sleep sessions as well as reduce consumption rate of a MS. PS allows an MS to sleep for a fixed duration and then listen for a fixed duration in a round-robin form. The PS reduced sleep sessions based on the type-2 PSC with QoS considerations. 


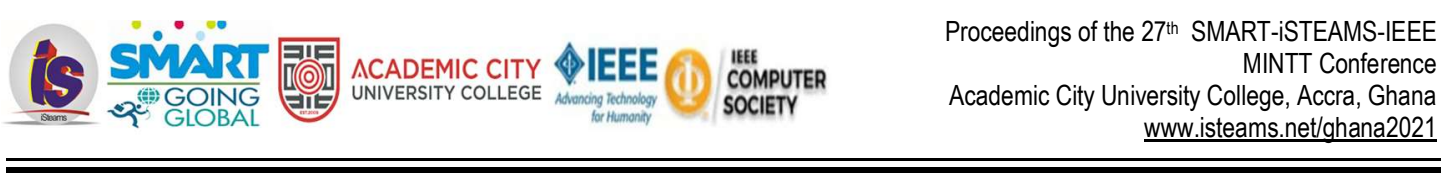

While the proposed AS on the other hand examine when a MS should sleep/stay awake. That is, the AS schedules resource to MS and inform MS to sleep/listen for every OFDM frame. AS implemented by type-3 PSC. The scheme, first sorts all connections on a MS according to their delay requirements, and assigns high priorities to these connections with tight delay requirements. The PS and AS Schemes increase more sleep time and reduce consumption than the existing schemes. But they ignore increase delay as well as apply the scheduling schemes to a multiple MS environments and the global optimization from a BS point of view respectively. In (Feng et al. 2015), a POMDP-based Sleep Window Determination (PSWD) was proposed to extend the battery life of MS. The scheme stochastically determines the adequate length of each sleep window based on traffic arrival. The scheme control the sleep cycles, with each control cycle overlapped with the adjacent one. The first control cycle begins at the last frame of MSs idle time in the normal session. The remainder control cycles are individually started at the exits of every listening interval with the previous control cycle. The proposed scheme significantly conserves energy better than the existing ones with increase in signaling overhead. In (Shi et al. 2006), a Longest Virtual Burst First (LVBF) scheduling algorithm was proposed to improve the battery life.

The LVBF scheme, schedule packets of MS in a virtual burst with one primary MS and a multiple secondary MS sharing the wireless link resource. In the proposed scheme, the primary MS has higher priority than the secondary MS in resource allocation, such that almost all the packets of the primary MS are transferred in the burst duration. As a result of burst broadcast, the LVBF scheme prolongs MS life by reducing the response time when MS Stay in the idle state and the number of MS transitions from wake/sleep interval. The proposed scheme achieved significant savings with QoS requirements at the expense of an increase in delay. In (Shih et al. 2018), a Wake on wireless technique scheme to enhance battery life of Personal Digital Assistants (PDAs) was proposed.

The scheme shut down the device and the wireless network card when not being used the device is powered only when an incoming call is received, Thereby increasing the battery lifetime. The scheme provided life improvements over other technologies. However, applications such as voice communications do not behave well under the proposed scheme; more so, there is an increase in delay of mobile device.

In (Lee et al. 2007), a Probabilistic Sleep Interval Decision (PSID) algorithm was introduced to examine MS sleep time, the scheme examines each Tj by considering the fixed and variable delays. The algorithm makes MS wake up intensively when the probability that a response packet will arrive at the BS is high. The algorithm calculates the target probability from the mean and standard deviation of $Y$, and examine the extension-allowed interval from the target probability, determine the number of sleep intervals to be included in the extension-allowed interval, divide the extension-allowed interval into subintervals by using the CDF of $Y$ and outside the extension-allowed interval, the scheme repeat the last sleep interval included in the extension-allowed interval. it significantly decrease consumption and buffer delay than the BTE scheme with average response delay.

In (Ritesh et al. 2009), a novel Sleep-Mode Manager Architecture was proposed to negotiate the sleep duration of MS, and compute the sleep-time per QoS, by aggregating the unavailability periods of all the active PSC_ID traffic. The architecture reduce the overhead of management messages enabling the architecture to conserve energy and bandwidth at the same time support dynamic adaptation in sleep-mode parameters. The architecture outperformed the standards in conserving energy and bandwidth. It however ignores combining algorithms to classify connections them into PSC along with joint PSC managements. 


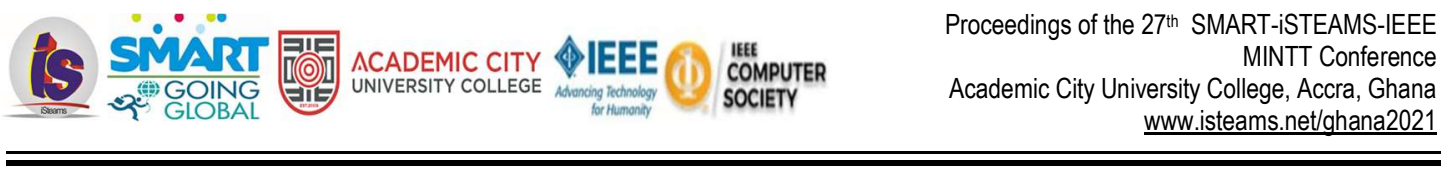

In (Yang et al. 2010), a Load-Based Power Saving (LBPS) Aggr scheme was introduced to improve efficiency. The scheme measures the traffic load and estimates the sleep window size for MS by setting a proper threshold for data accumulation threshold. The scheme significantly improved efficiency better than Type I and II at the cost of slight response delay.

\subsection{Adaptive Power Saving Mechanisms}

In (Min-Gon et al. 2008), an Adaptive Power Saving Mechanism (APSM) was proposed to extend the battery life. The scheme adaptively adopts the sizes of Tmin and Tmax by considering the request period of each initiation of awakening ( $T$ initial) in the previous sleep-mode duration. During each sleep-duration, when there is no initiation of awakening in each sleep cycle, the size of operating sleep interval doubles from the size of the previous sleep interval until Tmax, when an initiation of active state occurs, Tmin and Tmax are updated based on the following policies namely: (1) when Tf is equal to Tmin and Tmin is smaller than Tmax, then Tmax is regarded as a relatively larger size compared to Tmin.

Thereby, Tmax is updated and becomes half of the Tmax in the previous operation. (2), when Tf is equal to Tmax and Tmax is smaller than TMAX, Tmax is then regarded as a relatively smaller size compared to Tmin and the Tmax doubles. (3) Else, Tmin is updated to half of Tf. The scheme decrease the number of sleep cycles with increase response time. In (Mugen and Wenbo, 2008), an Adaptive Energy Saving Mechanism (AESM) was proposed to examine the sleep intervals considering traffic load and MS requirements.

The scheme adaptively examine the initial sleep interval and the period sleep interval according to $Y$ and $\Psi$ parameters, The scheme sets between efficiency as well as delay. But ignores examining the traffic load threshold as well as enhancing the AESM. In (Jenhui et al. 2014), a novel downlink (DL) and uplink (UL) Alignment (DUAL) scheme was proposed to enhance efficiency of MS. The proposed DUAL scheme used the frame arrival rate of UL $(\lambda \mu)$ and a safe threshold of buffer size QT as the parameters to estimate the maximum allowable waiting time to align the UL with the $\mathrm{DL}$ connections. The mechanism extends MS energy conservation when UL traffic is greater than DL traffic with increase consumption.

In (Seungkwon and Youngil, 2007), an adaptive initial-sleep window scheme was proposed to decrease consumption rate. The proposed scheme updates old initial-sleep window (W1 old), with the new initial window (W1 New) when the MS ends sleep mode operation. keeps track of TB as well as trie to adaptively make the sum of $\mathrm{W} 1$ and $\mathrm{T}$ be equal to TB. The value of $\sigma$ parameter adjusts the speed of adaptation; while the scheme allows MS to stay in sleep state as long as possible in burst arrival duration. The scheme utilizes the previously measured TB to decide the New. It improved efficiency in terms of wake and normalized duration, with increase in response time.

In (Liu et al. 2011), a novel counter-driven adaptive sleep mode scheme was proposed to decrease signaling overhead and balance power consumption and latency. It ensures that the length of the sleep cycle is adjusted adaptively to enhance effectiveness according to the change of user activity level simultaneously decreasing signaling overhead. The size of the sleep window is decided in real-time by traffic activity level true tracking counters. The scheme reduced signaling overhead and balanced efficiency as well as delivery latency. But ignores parameterization for different data traffic types and their combinations. In (Jin et al. 2011), an Adaptive Sleep Mode Management (ASMM) scheme was proposed to minimize consumption of MS. The scheme adjusts MS sleep cycle and listening intervals in an adaptive manner based on online monitoring and estimation of the traffic condition. 


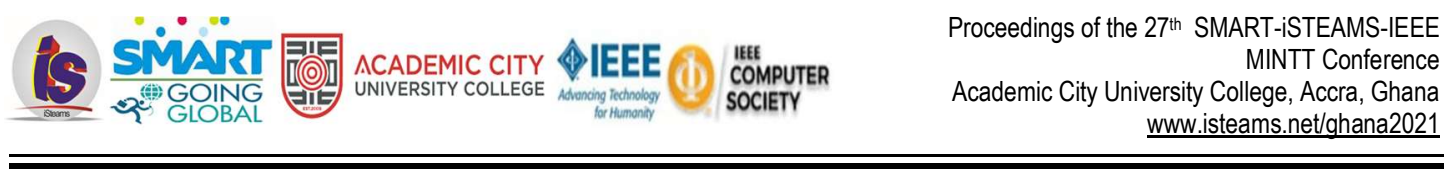

The scheme monitors DL frames for each MS and estimates the frames arrival rate online enabling the scheme to adjust the sleep cycle for the MS and use the AAI-SLP REQ signaling message to notify the station of the new sleep cycle length. The proposed scheme decreased consumption of MS with a userspecified packet delay constraint. With challenges of operational performance under a bur-sty and heavy traffic load and signal overhead. In (Sanghvi et al. 2008), an adaptive waiting time threshold algorithm was proposed to minimize consumption.

The scheme dynamically adjusts the idle threshold based on $\mathrm{DL}$ and $\mathrm{UL}$ traffic arrival to predict best duration of response threshold. The idle threshold is determined by using various weighting factors arrival rate and the previously arrived rate. It is chosen to be small during low-traffic load to allow the MS to switch to sleeping mode without additional delay while chosen to be large during heavy traffic to keep MS in an active duration to minimize the sleep/wake duration. The scheme reduced the consumption of MS especially under low traffic, at the expense of an increase in response delay.

In (Hsu and Feng, 2010), an Adaptive Listening Window (ALW) scheme was proposed to improve battery performance. The scheme dynamically tunes the length of the listening window according to the number of both arrival and retransmission packets and the delay constraint. In the scheme, the length of each listening window is adaptively adjusted based on the total number of buffered packets that exist in the arrival buffer and retransmission buffer as well as the delay constraint. The arrival buffer is utilized to preserve the arrival packets in the previous sleep cycle, whereas the retransmission buffer keeps packets that are ready to be retransmitted within the current listening window. The listening window is tuned increased/decreased for each sleep cycle. It diminished packet loss rate and save battery life with minimal packet loss rate for RTVR connections.

While (Lee et al. 2007), an adaptive sleep mode interval control algorithm was proposed to reduce consumption. The scheme adapts Tmin according to DL traffic pattern to predict the next arrival of the DL frame. These cuts the number of listening intervals in the sleep duration as well as reduce consuming power of MS in sleep state, as the sleep interval approaches Tmax; is incrementally increased as an average of nth sleep interval and Tmax. Thus, decreasing the delay, DL frame occurs waiting for the MS to wake up. The scheme improved the amount of consumption at lower traffic as well as at higher traffic but no consideration for uplink traffic.

In (Sanghvi et al. 2008), an adaptive waiting time threshold scheme was proposed to reduce consumption. The scheme adjusts the idle threshold according to $\mathrm{DL}$ and $U \mathrm{~L}$ traffic pattern to predict a better duration of waiting time. The idle threshold is determined using various weighting factors to the recently arrived packet rate and the previously arrived rate. The scheme selects wisely during low-traffic load to allow the MS to switch to sleep mode without any further delay while selecting large during heavy traffic to keep MS in an active state in order to reduce the sleep-wake session. The scheme decrease consumption under low traffic at the expense of an increase in waiting time. 


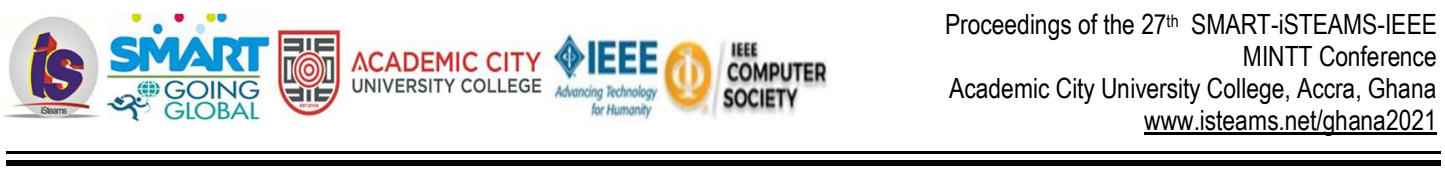

\subsection{Dynamic Power Saving Mechanisms}

In (Jianbin et al.2008), a dynamic traffic load-aware scheme was proposed to increase efficiency of a battery life of MS. The scheme used a dynamic scheme to tune the idle check time based on the statistical measurement of traffic load. The scheme enhanced as well as decreased the mean response time as compared to the existing PS scheme with increase in the complexity. In (Zhu et al.2007), a heuristic algorithm was proposed to decrease delay and the rate of consumption.

The scheme tunes the sleep parameters based on traffic load and response requirements. The scheme succeeded in bounding the response time with increase in consumption. While in (Chang et al. 2012), a Dynamically Alternating Sleep Interval Scheduling Algorithm (DASISA) was proposed to schedule series of PSC. It schedules more proper queue based on frame arrival. As well as examine the sleep mode adjustments, with listening intervals interleaved with the processing frames. Based on frame arrival as well as established a trade-off for consumption and response time. The proposed DASISA improved battery life at the expense of unnecessary response delay.

In (Xue et al. 2011), a dynamic scheme was proposed to enhance battery efficiency of MS. The proposed scheme tunes the ratio of the sleep duration and receives frames according to their arrival load. The scheme enhanced efficiency with increase response duration. In (Kwon et al. 2010), a dynamic power saving scheme was introduced to increase efficiency of MS, the MS used PSC to adjusts the MS sleep intervals of the sleep mode of PSC I in order to match the start point of the listening intervals of PSC I with the nearest listening window of PSC II. In PSC I the size of the sleeping is double size of previous ones subsequently. While in of PSC II the size of the sleep window is fixed due to UGS. Hence, the sleep interval of PSC I was adjusted. The scheme reduce consumption rate of MSs with no consideration for the adjustments of PSC II.

In (Chen et al. 2009), a Maximum Unavailability Interval (MUI) scheme was proposed to enhance energy efficiency for Type II. The MUI dynamically adjust the start frame number of each PSC to enable efficiency of the sleep intervals. The scheme examines the number of frames at the unavailability interval using Chinese Remainder Theorem, when the sleep cycle length is pair-wise as well as relatively prime, all the two main parameters as $n$ and $x$ are found. Such that, when the length of sleep cycle $a s m_{i}$ is not pair-wise as well as prime, then the existence of $n$ solution of $x$ is no longer assured. It reduced consumption. But, ignores considering how to map QoS conditions into the parameters of PSC as well as proposed a scheduling scheme, hence, minimizing the unavailability interval with improved QoS.

While in (Kwon et al. 2009), a Dynamic scheme (DPSM) was proposed to increase efficiency of MS. The scheme tunes the sleep intervals of PSC I (III) to match the starting point of listening interval in PSC I (III) with that of the listening intervals in PSC II. The schemes decreased the MS listening session as well as increase the unavailability interval. It reduced battery consumption of MS with an increase unessary response delay requirements which may result to loss of packets. In (Chou et al. 2013), a Battery LifetimeAware Power Saving Scheme (BLAPS) was proposed to improve battery life performance of MS. The proposed BLAPS dynamically tune the operating parameters based on the remaining battery life of MS and the traffic arrival with frequent switching to listening intervals which increased the rate of consumption. 
In (Liu et al. 210), a Dual scheme was proposed to balance power consumption as well as response delay requirements. The proposed scheme tunes the initial intervals according to a pre-defined MS threshold. The scheme decrease MS consumption with an increase in response MS. Finally, in (Priya et al. 2013), a Queuing model based Scheme (QPS) was proposed to enhance battery life of MS. The proposed QPS adjusts the sleep intervals for different traffic classes of PSC. While it sleep interval size of the sleep mode is examined based on frame arrival as well as type of traffic arrivals. It reduce the consumption rate with increase in delay. Power Saving Scheme (DAPSS) was proposed (Wisdom et al. 2019) to minimize the longer sleep intervals of MS.

The scheme successfully minimized the longer sleep intervals of MS; thereby, decreasing the response delay of MS while maintaining power savings respectively. However, the scheme ignores in-cooperating real time services, which may further improve on the overall performance of the MS.

In (Wisdom et al. 2019) an Enhanced Battery-Life power saving scheme (EBPSS) was proposed to incooperate real time services, which is an improvement of the existing DAPSS Scheme. The Scheme incooperates real time services and successfully extends the battery life performance with a little increase in consumption. (Saidu et al. 2017) Proposed a Hyper-Erlang Battery-Life Energy Scheme (HBLES) to analytically adjust the sleep parameters based on the remaining battery power and the traffic pattern to simultaneously decrease the ragte of MS consumption and delay. It uses a Hyper-Erlang distribution to determine the behavior of the traffic. The scheme improves the energy efficiency. However, it ignores uplink traffics.

\section{RESULTS AND DISCUSSION}

The findings of this research are presented in table 1 , as well as a comparative analysis of these schemes below in 3.1-3.2 respectively.

\subsection{Comparison of Power Savings Classes (PSC)}

Table 1 presents a comparison of various algorithms. These algorithms are compared in terms of their standards; power saving classes, traffics, operational mode and QoS. While in Figure 2 and 3 an analysis of the percentage of proposed schemes is presented in order to efficiently explore the left behind areas especially in energy Savings. 
TABLE 2: Summary of the REVIEW

\begin{tabular}{|c|c|c|c|c|c|c|c|c|c|c|c|c|c|}
\hline \multirow[t]{2}{*}{ REF } & \multicolumn{2}{|c|}{ IEEE 802} & \multicolumn{2}{|c|}{ PSC } & \multicolumn{3}{|c|}{ Traffic } & \multicolumn{2}{|c|}{ Operation Mode } & \multirow[t]{2}{*}{ Algorithm } & \multirow[t]{2}{*}{ QoS } & \multirow[t]{2}{*}{ Strength } & \multirow[t]{2}{*}{ Weakness } \\
\hline & $16 e$ & $16 \mathrm{~m}$ & I & II & $\mathrm{DL}$ & UL & Model & Normal & Sleep & & & & \\
\hline [6] & & & & & & & $\mathrm{M} / \mathrm{G} / 1$ & & & TRF-IND & & $\begin{array}{l}\text { Minimize the } \\
\text { average power } \\
\text { consumption } \\
\text { and satisfy } \\
\text { QoS }\end{array}$ & $\begin{array}{l}\text { Ignore considering } \\
\text { the little increase } \\
\text { response delay }\end{array}$ \\
\hline$[7]$ & & & & & & & $\mathrm{M} / \mathrm{G} / 1$ & & & TRF-IND & & $\begin{array}{l}\text { Enhance } \\
\text { power saving } \\
\text { efficiency }\end{array}$ & $\begin{array}{l}\text { Complicated } \\
\text { equations } \\
\text { derivation } \\
\end{array}$ \\
\hline$[8]$ & & & & & & & Markov & & & TRF-IND & & $\begin{array}{l}\text { Improve power } \\
\text { saving \& \& } \\
\text { minimize delay }\end{array}$ & $\begin{array}{l}\text { An increase in the } \\
\text { algorithm } \\
\text { complexity }\end{array}$ \\
\hline
\end{tabular}

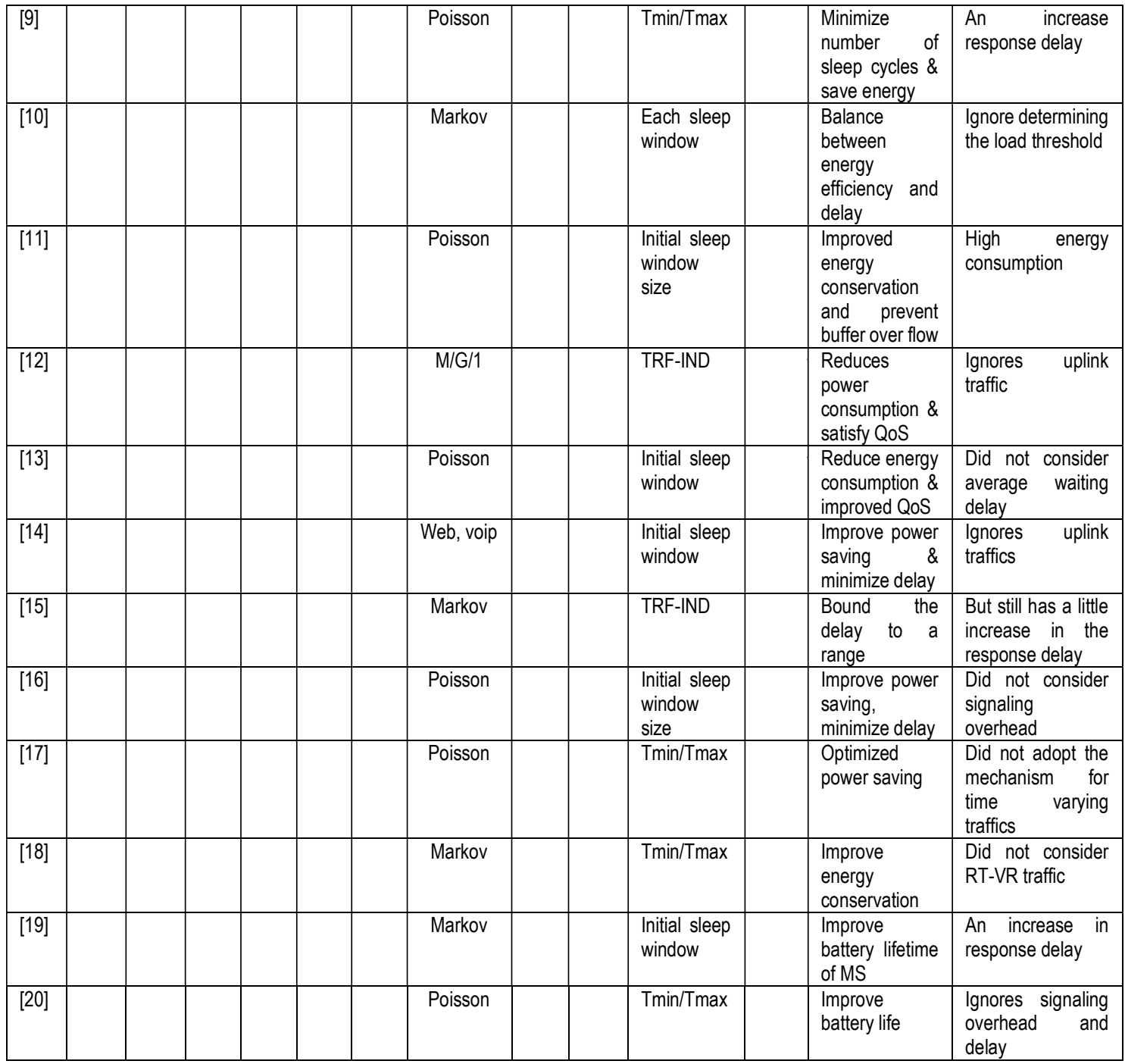




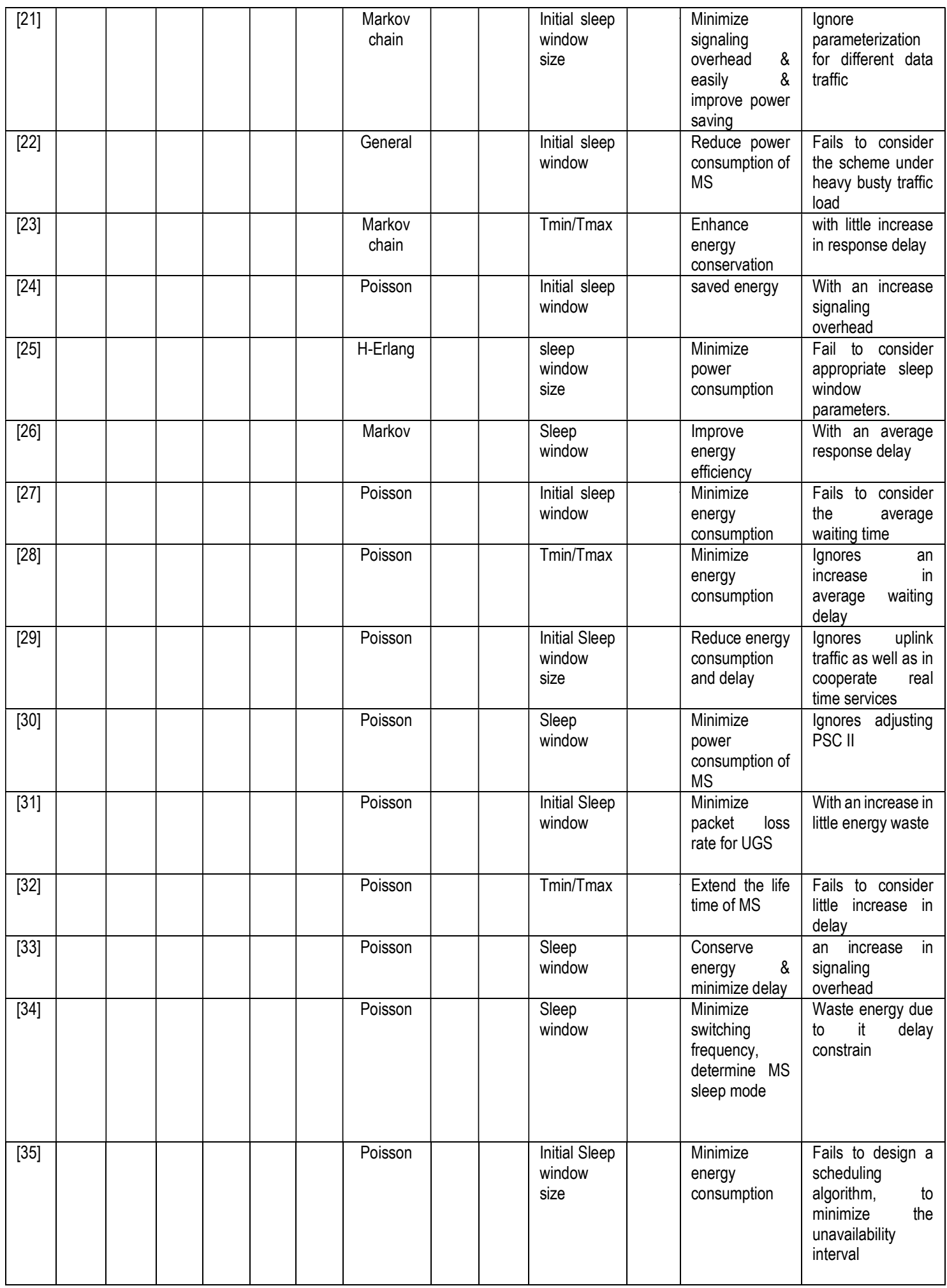




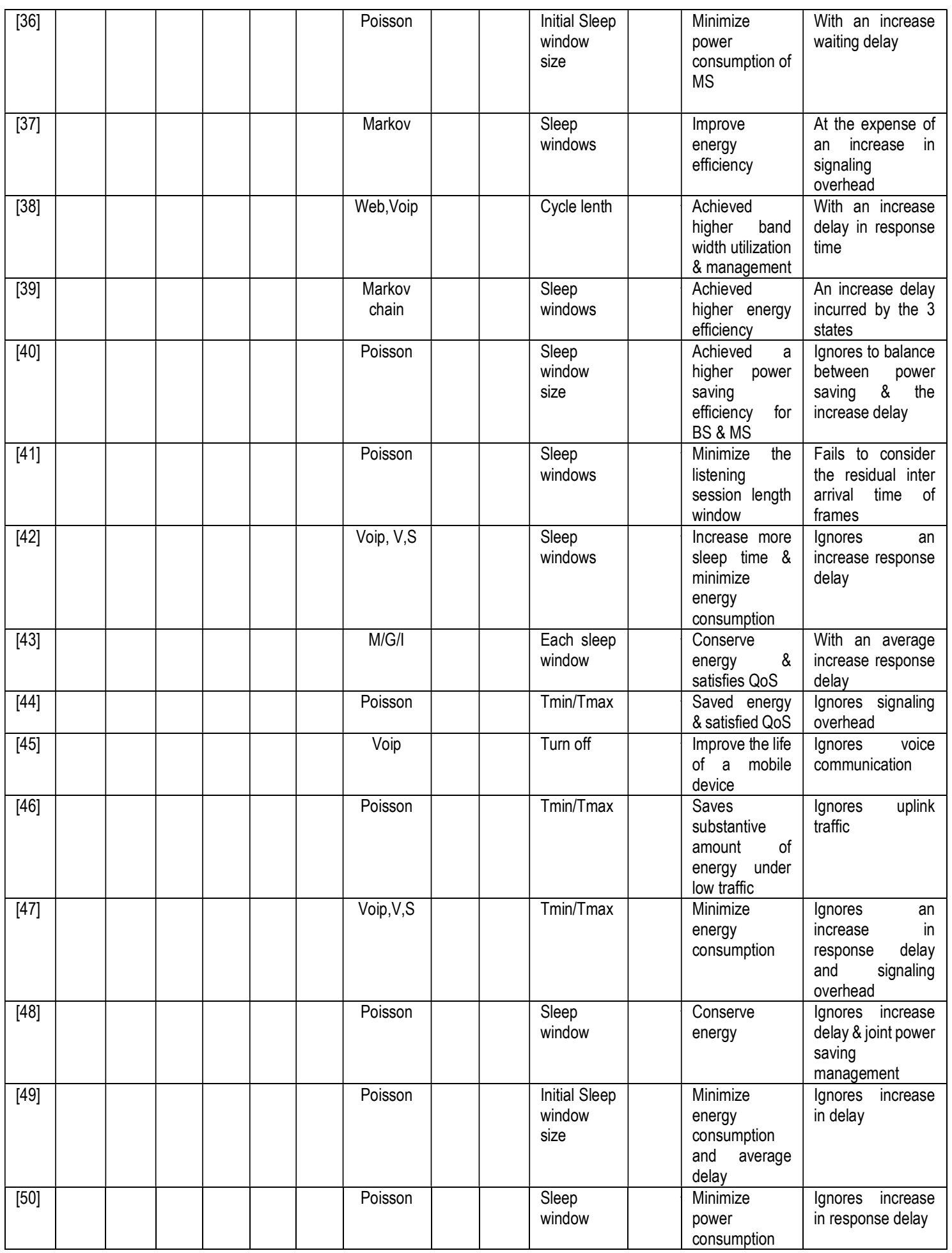




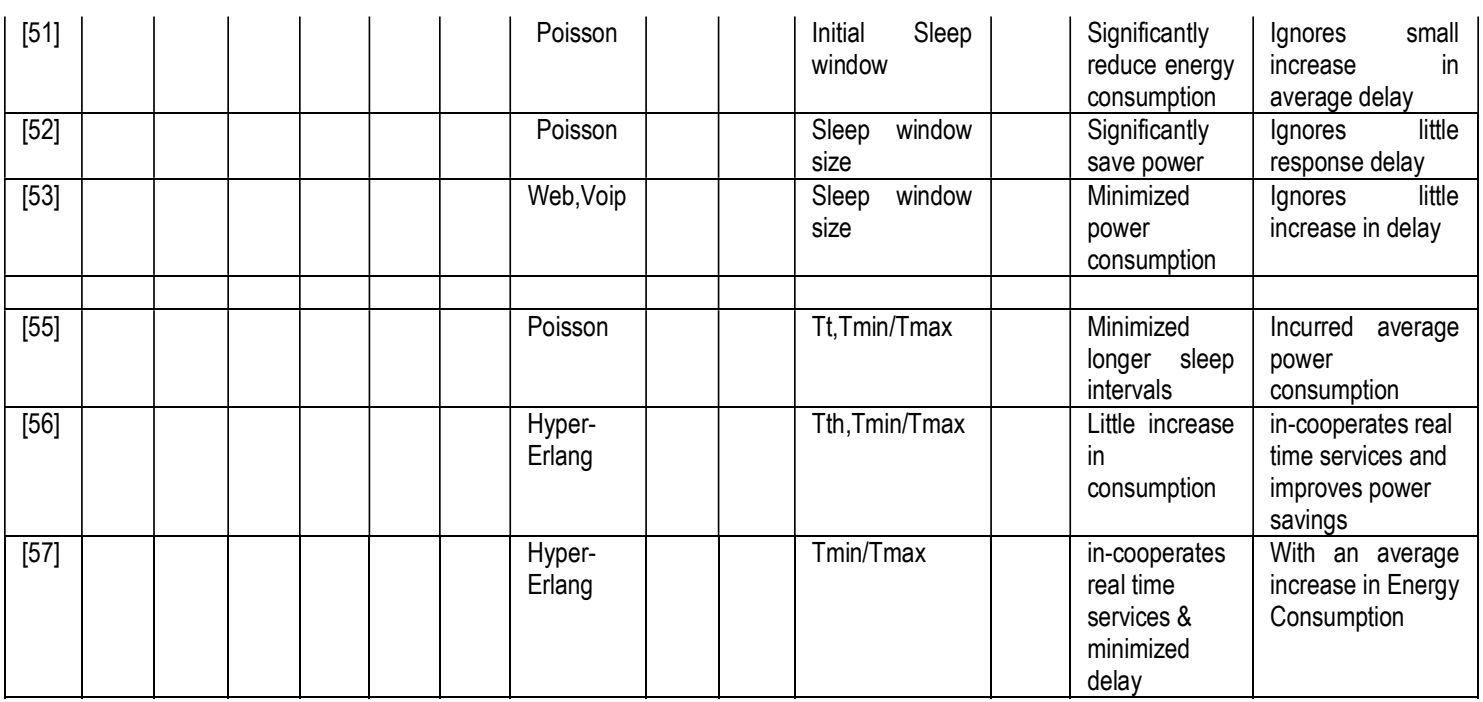

\subsection{Comparative Analysis}

In this section, we present a comparative analysis of the existing schemes as follows

[6]-[12][14]-[21][22]-[33][35]-[37][39]-[49][51]-[55] of the existing algorithms focused on deriving analytical models for IEEE 802.16e system; while [7][13][22][30][32][34][38][44][50] of the algorithms were on the most recent IEEE 802.16m standard. [1][3][4][11]-[55] models for downlink transmission while [11][21][23][25][26][29][35][44][46][50][53] models for uplink transmission. Sleep mode and operations were developed.

However, [11][21][23][25][26][34][43][48][51] of the Existing schemes focused on uplink transmission and [11][17][27][38][39][43][44] uses normal operational modes while [6]-[19][20]-[37][40]-[53] uses a sleep operational modes. More so, adaptive algorithms were proposed in some of the existing works in order to provide various decision policies, such as sleep window size, to achieve better performance under different traffic status. For example, "each sleep window" implies that the algorithm can adaptively adjust every sleep. 


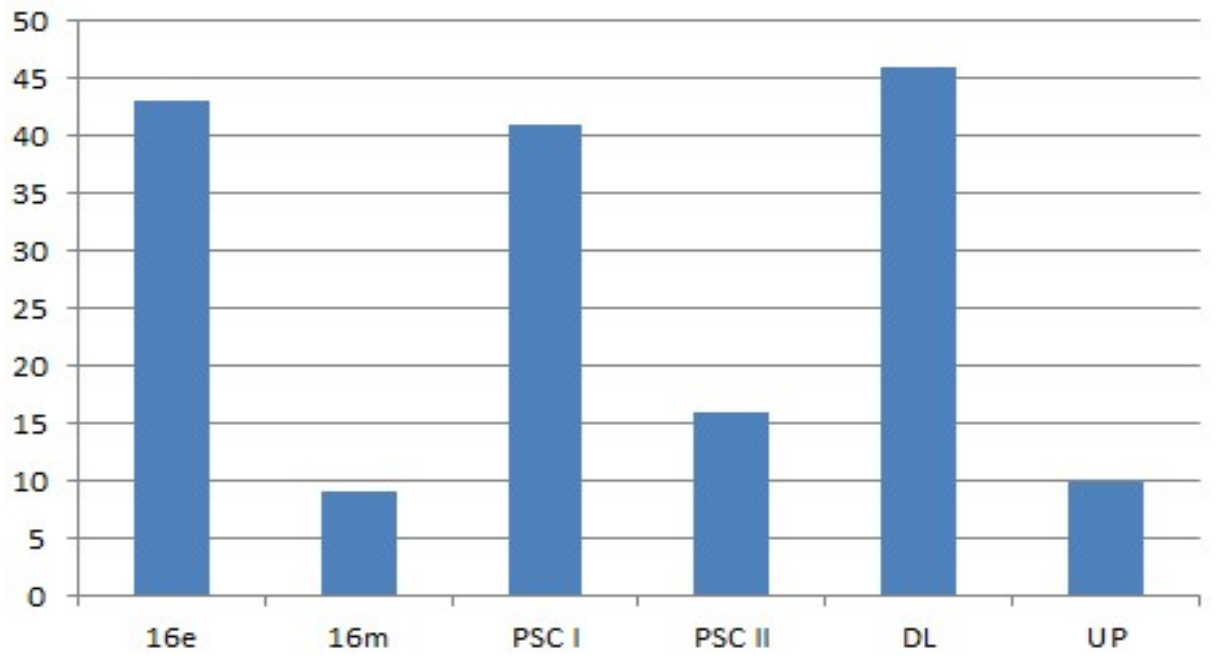

Figure 2. Shows an analysis and the percentage (\%) of IEEE $802.16 \mathrm{e} / \mathrm{m}$, power saving classes of both type I \& II and operations in both downlink and uplink traffics.

\section{Analysis of Modes}

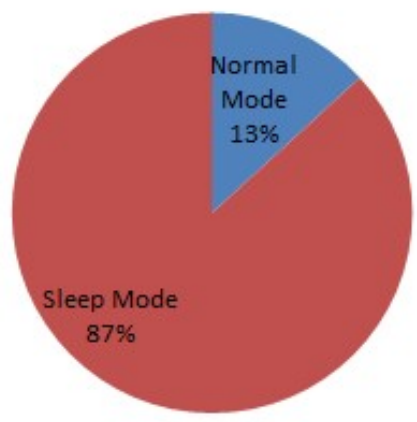

Figure 3: Shows the Analysis of Both Sleep and Normal Modes in PSC.

Window size, while that with the "initial sleep window size" can only adjust the initial sleep window size. The "state status" implies that the mechanism can determine which state the MS should switch to; including normal mode, PSC of Type I, and PSC of Type II. The "TRF-IND interval" implies that the algorithm can adjust the traffic indication interval, in which BS periodically sends Traffic indication messages to inform MS whenever there is a packet to receive or transmit. More so, the QoS requirement among all different schemes may correspond to packet delay constraint or spectrum efficiency. Note that, both sleep mode and normal mode are supported by IEEE $802.16 \mathrm{e} / \mathrm{m}$ standards. It is clear from the table That most of the existing studies only focused on the power saving in the period of sleep mode with downlink traffic for IEEE 802.16e system. Finally, we also compare their individual strength and weaknesses. The strength; implies the achievement of the researched paper while the weakness: implies the limitations of the paper and possible open issues for future research towards efficient power-saving of mobile devices. 


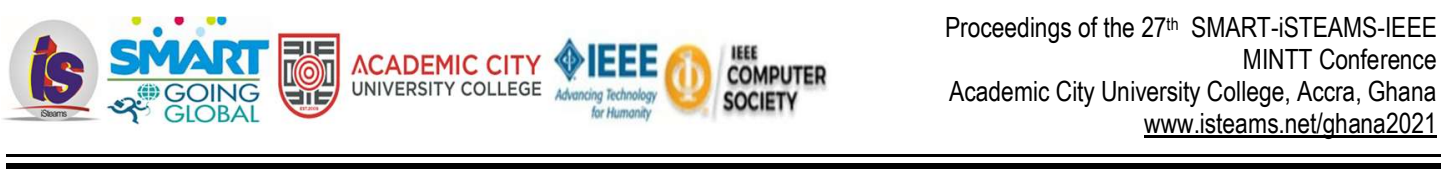

\section{CONCLUSION}

In this paper, a survey on energy-saving schemes in WiMAX is presented. In the survey, the operational procedure, strengths and weaknesses of each scheme were presented in details, in order to understanding how efficiency of MS battery life are enhanced via different strategies and ways in which some of the left over important problems in WiMAX could be addressed. In addition, a comparative analysis of these schemes is also presented. The analysis identified open issues for possible future directions towards efficient energy savings in IEEE 802.16e WiMAX Networks.

\section{REFERENCES}

1. Almhana J, Liu Z, Li C and Mc-Gorman R (2008). Traffic Estimation and Power Saving Mechanism Optimization of IEEE 802.16e Networks. Proceedings of the IEEE ICC, 19(2) 2008. [17]

2. Azad A.P, Alouf $S$ and Altman E (2011). Optimal Control of Sleep Periods for Wireless Terminals. IEEE Journal on Selected Areas in Communications, 29(8), SEPTEMBER 2011. [41]

3. Chang $J$ and Lin $Y$ (2012). Dynamically Alternating Power Saving Scheme for IEEE 802.16eMobileBroadbandWirelessAccessSystems, Journalofcommunications and Networks, 14(2), APRIL 2012. [16]

4. Chen T-C, Chen J-C and Chen Y.Y (2009). Maximizing Unavailability Interval for Energy Saving in IEEE 802.16e Wireless MANs. 8 (4), APRIL 2009. [35]

5. Chou L.D, Li D. C and Hong W.Y (2013). Improving energy efficient Communications with a battery lifetime-aware mechanism in IEEE 802. 16eWireless networks, Concurrency and Computation: Practice and Experience, (25), pp. 94-111. [49]

6. Chen Y-L, Tsao S-L (2006).Energy-efficient Sleep-mode Operations for Broadband Wireless Access Systems. IEEE 64th Vehicular Technology Conference, 2006. Pp 1-5. [42]

7. DRAFT Amendment to IEEE Standard for Local and Metropolitan Area Networks - Part 16: Air interference for Fixed Broadband Wireless Access Systems - Advanced Air Interface, IEEE P802.16m/D7, July 2010. [4]

8. Eunju H, Kyung J.K, Jung J. S and Bong D. C (2007). The Power Saving Mechanism with Periodic Traffic Indications: A New Sleep Mode Scheme in the IEEE 802.16e. Proceedings of the Third Korea-Netherlands Conference on Queueing Theory and its Applications to Telecommunication Systems. 2007, pp. 319-334. [12]

9. Eunju H, Kyung J. K, Jung J. S and Bong D. C (2009). The power saving mechanism with binary exponential traffic indications in the IEEE $802.16 \mathrm{e} / \mathrm{m}$. Queuing Systems: Theory and Applications, 62 (3), pp. 197-227, July 2009. [7]

10. Eunmi C. N-O and Dan K. S. (2015). Remaining Energy-Aware QoE Based Binary-Exponential Sleep Mode Operation in IEEE Communication Letters 19(12), DECEMBER 2015. [13]

11. Feng K-T, Wun-Ci S and Chun-Yu C (2015).Comprehensive Performance Analysis and Sleep Window Determination for IEEE 802.16 Broadband Wireless Networks. IEEE Transactions on Mobile Computing. Pp1536-1233. [5]

12. Ferng H.W and Li H.Y (2013). Design of predictive and dynamic energy efficient Mechanisms for IEEE 802.16e, Wireless Personal Communications, 68 (4), pp. 1807-1835.

13. Gary K.W, Zhang Q and Tsang D-H. K (2009). Switching Cost Minimization in the IEEE 802.16e Mobile WiMAX Sleep Mode Operation. (9) June 21 - 24, 2009. 


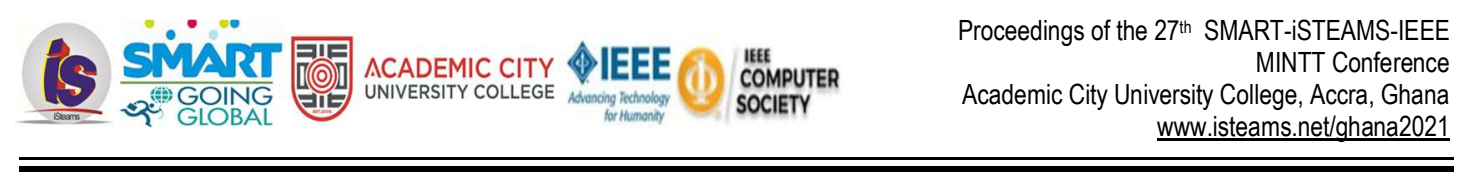

14. Hwang E, Kim K. J, Son J. J and Choi B. D (2010). The Power-Saving Mechanism with Periodic Traffic Indications in the IEEE 802.16e/m. IEEE Transactions on Vehicular. Technology, 59 (1), pp. 319-334, Jan. 2010. [6]

15. Hsu C-I-I and Feng K-T (2010). Performance enhancement and analysis for IEEE 802,16e/m sleep mode operations with unsolicited grant service/real-time variable-rate connections, IEEE Transactions on Microwave Theory and Application, 4 (9), 2010. pp. 1083 -1094. [31]

16. Hsein H-K, Feng K-T and Chang C-J (2010). Statistical Control Approach for Sleep-Mode Operations in IEEE 802.16m Systems. IEEE Systems Journal, 59(9), NOVEMBER 2010. [33]

17. Hsu C-H and Feng K-T (2009). A Statistical Power-Saving Mechanism for IEEE 802.16 Networks. 20TH IEEE International Symposium on personal, indoor and mobile radio communications, 2009. [37]

18. IEEE 802.16 WG (2004). Standard for Local and Metropolitan Area Networks Part 16: Air Interface for Fixed Broadband Wireless Access Systems, IEEE 802.16 Working Group and others, IEEE Std, 802.16. [1]

19. IEEE 802.16e WG (2005). IEEE Standard for Information Technology- Telecommunications and Information Exchange between Systems - LAN/MAN Specific requirements, Part 16: Air Interface for Fixed and Mobile Broadband Wireless Access Systems, IEEE Std802.16e. [2]

20. IEEE Standard for Local and Metropolitan Area Networks - Part 16: Air Interference for Fixed Broadband Wireless Access Systems, IEEE Standard 802.16-2009, May 2009.[3]

21. Jenhui C, Woei-Hwa T and Jiann-Der I (2014). A Downlink and Uplink Alignment Scheme for Power Saving in IEEE 802.16 Protocol. Scientific World Journal Volume Article ID 217973,11 pages. doi.org/10.1155/2014/217973. [11]

22. Jianbin X, Zhanting Y and QiuYu Z (2008). Traffic Load-Aware Power-saving Mechanism for IEEE 802.16e Sleep Mode, in College of Computer and Communication Lanzhou University of Technology Lanzhou, China. 2008, pp. 735-745.[8]

23. Jang J, Han K and Choi S (2006). Adaptive Power Saving Strategies for IEEE 802.16e Mobile Broadband Wireless Access II IEEE Communications, (APCC '06). Asia-Pacific Conference PP 124-128.

24. Jufeng X, Zou S, Ren B and Cheng S (2006). WLC17-6: An enhanced energy saving mechanism in IEEE 802.16 e, in IEEE Global Telecommunications Conference (GLOBECOM'06), pp.1-5.

25. Jin S, Chen X, Qiao D and Choi B. S (2011). Adaptive sleep mode management in IEEE $802.16 \mathrm{~m}$ wireless metropolitan area networks. In Computer Networks, 55 (16), pp 2642-2645. [22]

26. Kwon S and Cho D (2010). Enhanced Power Saving Through Increasing Unavailability Interval in the IEEE 802.16e Systems, IEEECommunications Letters, 14(5), JANUARY 2010. [30]

27. Kwon S-W and Cho D-H (2009). Dynamic Power Saving Mechanism for MS in the IEEE 802.16e Systems. In Communication and Information Systems Lab.School of EECS, KAIST Guseong-dong Yuseong-gu Daej eon, Korea, pp 373-381 2009. [36]

28. Kim M, Choi J. Y and Kang M (2009). Enhanced Power-Saving Mechanism to Maximize Operational Efficiency in IEEE 802.16eSystems. Elsevier 8(9), SEPTEMBER 2009. [23]

29. Kong L, Wong G. K. W and Tsang D. H. K (2009). Performance Study and System Optimization on Sleep Mode Operation in IEEE 802.16e.IEEE Transactions on Wireless Communications 8(9), pp 4518-4528 (2009) 11. [39]

30. Lee J-R and Cho D-H (2007). Performance Evaluation of Energy-Saving Mechanism

31. Based on Probabilistic Sleep-Interval Decision Algorithm in IEEE 802.16e. IEEE Transactions on Vehicular Technology, 56 (4), July 2007. [47] 


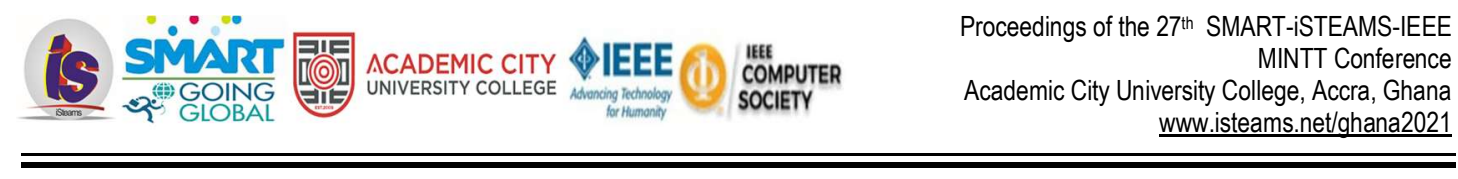

32. Liao W-H and Yen W-M (2009). Power- saving scheduling with a QoS guarantee in a mobile WiMAX system, II Journal of Network andComputer Applications, 32 (6), pp. 1144-1152.

33. Liao W-H, Liu S-C and Liu K. C (2016), Efficient scheduling algorithm for multiple mobile subscriber stations with unsolicited grant service in an IEEE 802.16e network, Journal of Network and Computer Application, Volume 63, PP 50-55. [38]

34. Lin Y.W and Wang J.S (2013). An Adaptive QoS Power Saving Scheme for Mobile WiMAX, Wireless Personal Communications, 69 (4) pp. 1435-1462.

35. Liu J, Ren F and Lin C (2010). A dual-threshold power saving mechanism in WiMAX. In IEEE/ACIS 9th international conference on computer and information science (ICIS) pp. 465-470. [50]

36. Liu E, Zhang J and Ren W (2011). A Counter-Driven Adaptive Sleep Mode Scheme for 802.16e Networks. 73rd IEEE Vehicular Technology Conference, Budapest, HU, pp 15-18 May, 2011. [21]

37. Lee J-R and Cho D-H (2007). Performance Evaluation of Energy-Saving Mechanism Based on Probabilistic Sleep Interval Decision Algorithm in IEEE 802.16e, IEEE Transactions on Vehicular Technology 56 (4), July 2007.

38. Mai Y.T, Yang C. C, Chen J. Y and Lin M. H (2012). An Integrated Load based power Saving Scheme in IEEE 802.16e, in Proc. the 2012 FTRA International Conference on Advanced IT, engineering and Management (FTRAAIM 2012), Seoul, Korea, Feb. 6-8, pp. 71-72

39. Mehta S, Seth N, Sharma N and Snigdha T (2013). A Novel Approach for Minimizing the Delay and Load in Wireless Network (WIMAX). Shilpa Mehta et al Int. Journal of Engineering Research and Applications www.ijera.com ISSN: 2248-9622, 3 (6), Nov-Dec, pp.1344-1350.

40. Min-Gon K, Kang M and Choi J. Y (2008). Remaining Energy-aware power Management mechanism in the 802.16e mac, in 5th IEEE Consumer Communications and Networking Conference (CCNC'08), pp. 222-226.

41. Min-Gon K., JungYul C and Minho K. (2008). Adaptive Power Saving Mechanism Considering the Request Period of Each Initiation of Awakening in the IEEE 802 Systems, IEEE Communications Letters, 12(2) pp106--108. [9]

42. Mugen $p$ and Wenbo W (2008). An Adaptive energy-saving mechanism in the wireless packet access networks. IEEE Communications Letters 12(4) pp 304-306 (2008). [10]

43. Nga D. T. T, KIM M and Kang M (2007). Delay-guaranteed Energy Saving Algorithm for the Delaysensitive Applications in IEEE 802.16e Systems. 2007. IEEE Transactions on Consumer Electronics, pp 534-541. [32]

44. Obaidat M.S and Boudrga K (2018). Border Surveillance with Wireless Sensor Networks (WSN) Systems in a distributed manner, IEEE Systems Journal 12 (4), 2018.

45. Priya M. D, Valarmathi M. L and Deepa M (2013). QPS: Queuing Model Based Power Saving Scheme For IEEE 802.16e Networks. International Journal of Science, Engineering and Technology Research (IJSETR), 2(5), May 2013. [53]

46. Ritesh K. K, Mayank R and Das D (2009). A Novel Architecture for IEEE 802.16m Subscriber Station for Joint Power Saving Class Management, (COMSNETS'09) Proceedings of the First international conference on communication Systems And Networks, Bangalore, India. January 05 - 10, 2009, PP 286-295. [48]

47. Saidu I, Subramaniam S, Jaafar A and Zukarnain Z. A (2014). Performance Analysis of WiMAX Networks: Discrete Event Simulator (DES) Development. International Review on Computers and Software. 9(11). Pp 1822-1833 


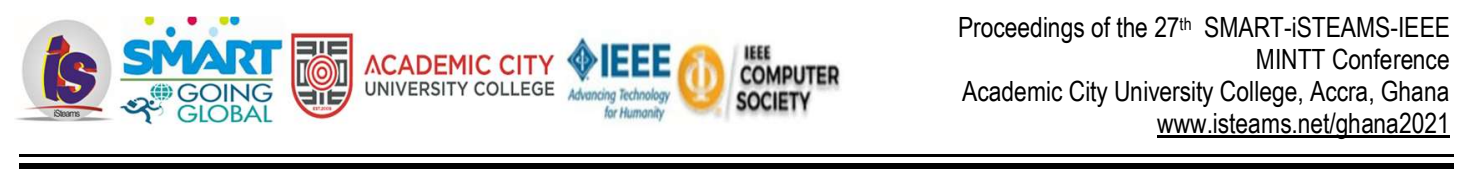

48. Saidu I, Azmi S and Zukarnain Z (2015). An efficient battery lifetime aware power saving (EBLAPS) mechanism in IEEE 802.16 e networks. Wireless Personal Communications (80), pp. 29-49. [29]

49. Saidu I, Musa H, Lawal M. A and Kane I. L (2017). Hyper-Erlang Battery-Life Energy Scheme in IEEE 802.16e Networks, Covenant Journal of Informatics \& Communication Technology 5(2), pp. 71-78. [57]

50. Sanghvi K, Jain P. K, Lele A and Das D (2008). Adaptive waiting time threshold estimation algorithm for power saving in sleep mode of IEEE 802.16e, in 3rd IEEE International Conference on Communication Systems Software and Middleware and Workshops (COMSWARE '08), pp. 334-340. [20]

51. Shi J, Fang G, Sun Y, Zhou J, Li Z and Dutkiewicz E (2006). Improving mobile station energy efficiency in IEEE 802.16e WMAN by burst scheduling, IEEE Globecom Conference, 2006. Pp15. [4]

52. Shengqing Z, Xiaoyu M and Lujian W 4(2007). A delay-aware auto sleeps mode operation for rower saving WiMAX. In Proceedings of 16th international conference on computer communications and networks, 2007 (ICCCN 2007) pp. 997-1001.

53. Seungkwon C and Youngil K. (2007). Improving Power Savings by Using Adaptive Initial-sleep Window in IEEE8O2.16e, IEEE Vehicular Technology Conference 65; 2007. [14]

54. Shih E, Bahl P and Sinclair M. J (2018). Wake on Wireless: An Event Driven Energy Saving Strategy for Battery Operated Devices, MOBICOM ACM, Aug 01, 2018. [45]

55. Vatsa O. J, Raj M, Ritesh K. K, Panigrahy D and Das D (2007). Adaptive power saving algorithm for mobile subscriber station in $802.16 \mathrm{e}$. In the $2^{\text {nd }}$ IEEE International conference on communication systems software and Middleware, 2007 (COMSWARE 2007), pp. 1-7. [46]

56. Wisdom D. D, Tambuwal A.Y, Mohammed A, Audu A, Soroyewun M. B and Isaac S (2019). A Delay Aware Power Saving Scheme (DAPSS) Based on Trafic load in IEEE 802.16eWiMAX Networks, Institute of Electrical and Electronic Engineering Conference (IEEE) Amadu Bello University Zaria, Kaduna State, Nigeria, $14^{\text {th }}$ to $17^{\text {th }}$ October 2019. [55]

57. Wisdom D.D, Tambuwal A. Y, Saidu I, Magami S and Elijah Y (2019). An Enhanced Battery Life Power Saving Scheme in IEEE, 802.16e Networks, International Journal of Engineering Applied Science and Technology (IJEAST), Volume 4, Issue 7 October 2019.

58. Wong G. K. W, Zhang Q, Tsang D. H. K (2009). Switching Cost Minimization in the IEEE 802.16e Mobile WiMAX Sleep Mode Operation, in AMC Wireless Communications and Mobile Computing: Connecting the World Wirelessly Conference (IWCMC), Leipzig Germany (9) pp 21 - 24, 2009. [27]

59. Wong G. K.W, Zhang Q and Tsang D. H.K (2009). Joint Optimization of Power Saving Mechanism in IEEE 802.16e Mobile WiMAX. in Proceedings of the IEEE GLOBECOM 2009. [34]

60. Xiao Y (2006). Performance Analysis of an Energy Saving Mechanism in IEEE 802.16 e wireless MAN, in 3rd IEEE Conference on Consumer Communications and Networking (CCNC'06), (1), pp. 406-410.

61. Xue J, Yuan Z and Zhang Q-Y (2008a). Traffic load-aware power-saving mechanism for IEEE 802.16 e sleep mode, in 4th IEEE International Conference on Wireless Communications, Networking and Mobile Computing (WiCOM'08), pp. 1-4.

62. Xue J, Yuan Z and Zhang Q-Y (2008b). Traffic Load-Aware Power-saving Mechanism for IEEE 802.16e Sleep Mode. College of Computer and Communication Lanzhou University of Technology Lanzhou, China, pp 1-7 


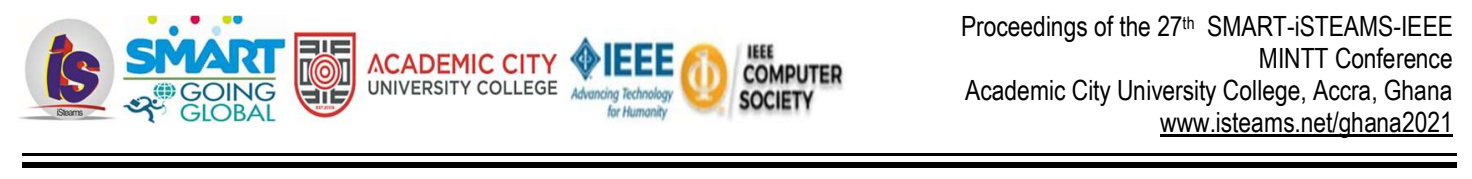

63. Xiao $Y$ (2005). Energy saving mechanism in the IEEE 802.16e wireless MAN, IEEE Communications Letters, 9(7), pp. 595-597. [24]

64. Xiao J, Ren S. Z. B and Cheng S (2006). An Enhanced Energy Saving Mechanism in IEEE 802.16e in 2006 31st IEEE Conference on Local Computer Networks; 2006. PP 1-5. [18]

65. Xue J, Yuan Z and Zhang Q (2008). Traffic load-aware power-saving mechanism for IEEE 802.16 e sleep mode, in 4th IEEE International Conference on Wireless communication Networking and MobileComputing (WiCOM'08), pp. 1-4.

66. Xue J, Zhang T, Li S and Wang W (2011). A Dynamic Algorithm of Energy-saving Mechanism for Real-time Polling Service. In the Third International Conference on Multimedia Information Networking and Security: Shanghai, China. 2162-8998. [26]

67. Yang C. C, Fang C. H and Lin J. R (2010). Adaptive Power Saving Strategy Based on Traffic Load in the IEEE 802.16e Network, in Proc. International Conference on Information and Communication Technologies (ICICT 2010), pp. 26-28. [52]

68. Yang, C.-C, Mai Y-T, Chen J.-Y and Kuo Y-C (2013) Integrated load-based power saving for BS and $\mathrm{MS}$ in the IEEE 802.16e network, in wireless communications and mobile computing. International Journal of Future Computer and Communication, 1(83) 2013. [40]

69. Yang S-R (2007). Dynamic Power Saving Mechanism for 3G UMTS Systems. Department of Computer Science, National Tsing Hua University, Hsinchu, Taiwan, R.O.C in Mobile Networks and Applications. Pp 1-8.

70. Zhang $Y$ (2007). Performance modeling of energy management mechanism in IEEE $802.16 \mathrm{e}$ mobile WiMAX. In Wireless Communications and Networking Conference (WCNC 2007). IEEE, pp. 3205-3209. [57]

71. Zhu S and Wang T (2007). Enhanced power efficient sleep mode operation for IEEE $802.16 \mathrm{e}$ based WiMAX, in IEEE Mobile WiMAX Symposium, pp. 43-47. [19]

72. Zhu S, Ma $X$ and Wang $L$ (2007) A delay-aware auto sleep mode operation for Power saving WiMAX, in Proceedings of 16th International Conference on Computer Communications and Networks (ICCCN'07), pp. 997-1001. [15]

73. Zhang $Y$ and Fujise $M$ (2006). Energy management in the IEEE 802.16e MAC, IEEE Communications Letters, (10), pp. 311-313.

74. Zhu S and Wang T (2007). Enhanced power efficient sleep mode operation for IEEE $802.16 \mathrm{e}$ based WiMAX. In IEEE Mobile WiMAX Symposium, Orlando, FL, USA, pp. 43-47, 2007.

75. Zhang Y, Xiao Y and Leung V. C. M (2009). Energy Management Analysis and Enhancement in IEEE 802.16e Wireless MAN, IEEE Transactions on Vehicular Technology, 58(7), SEPTEMBER 2009. 